\title{
Modal Parameter Identification and Numerical Simulation for Self-anchored Suspension Bridges Based on Ambient Vibration
}

\author{
Bing LIU ${ }^{1}$, Li Guo SUN ${ }^{2}$ \\ ${ }^{1}$ Department of Civil Engineering, Nanjing Technical Vocational College, Nanjing, Jiangsu 210019, China \\ ${ }^{2}$ College of Mechanics and Materials, Hohai University, Nanjing 210098, China
}

\begin{abstract}
This paper chooses the Nanjing-Hangzhou high speed overbridge, a self-anchored suspension bridge, as the research target, trying to identify the dynamic characteristic parameters of the bridge by using the peak-picking method to analyze the velocity response data under ambient excitation collected by 7 vibration pickup sensors set on the bridge deck. The ABAQUS is used to set up a three-dimensional finite element model for the full bridge and amends the finite element model of the suspension bridge based on the identified modal parameter, and suspender force picked by the PDV100 laser vibrometer. The study shows that the modal parameter can well be identified by analyzing the bridge vibration velocity collected by 7 survey points. The identified modal parameter and measured suspender force can be used as the basis of the amendment of the finite element model of the suspension bridge. The amended model can truthfully reflect the structural physical features and it can also be the benchmark model for the long-term health monitoring and condition assessment of the bridge.
\end{abstract}

\section{Introduction}

The main cable of the self-anchored suspension bridge is anchored directly at both ends of the stiffening beam, and the horizontal force of the main cable and the vertical force of the anchor cable are directly supported by the stiffening beam. The self-balancing system is formed in the horizontal direction of the stiffening beam. In addition to its general ground anchor suspension bridge beautiful shape and good mechanical properties, it need not build the large volume of anchorage, which can be applied to poor geological conditions and urban areas without anchorage. However, since the anchorage point of the main cable is on the main beam, the strength, stiffness and stability of the main girder are critical to the structural safety of the full bridge. As the main force component, under the load of vehicle et al., the main beam is prone to undergone, crack, the effectiveness reduce of prestress and the overall stiffness reduce of the structure.Therefore, regular health monitoring of such bridges is an important method for ensuring the safe operation of the bridge during the design life. With the rapid development of structural health monitoring technology, more and more structures have established a health monitoring system $[1,2]$. Due to the complex structure of the bridge and the large number of external factors, there are many uncertainties. How to use the monitoring data of a limited number of sensors to evaluate the health status of the structure is an urgent problem facing the academic and engineering circles. With the increasing ability of large-scale numerical calculation, it is possible to establish a complex and realistic numerical model, and it is possible to simulate the structural response of large-scale civil structure under various working conditions [3, 4]. Under the condition that the simulated structural response and the actual monitoring data have good compatibility, the numerical simulation results can make up the deficiency of the actual monitoring data, and realize the damage identification and state assessment of structures. In order to achieve the above objectives, the primary task is to establish with a sufficient accuracy, reflecting the bridge dynamic characteristics and physical characteristics.

Because of the errors of the model, the error of the structural parameters and the simulation difficulties of the boundary conditions, the bridge structure response and the actual response will be different, even if the finite element model is established strictly according to the

* Corresponding author:67689704@qq.com 
design drawings. The model must be corrected and verified, in order to get the actual state of the structure of the benchmark finite element model [5]. So far, many researchers have used different methods to study the different bridge structures based on the measured data [6-8]. However, the structure of the self-anchored suspension bridge is rarely studied. Based on this, the initial finite element model of the bridge structure is established by using the large-scale finite element software ABAQUS, which is based on the Nanjing-Hangzhou high-speed cross-bridge and learning eight-way self-anchored suspension bridge. The finite element analysis model of the bridge structure under the working environment was modified by the measured structural response, the internal force of the boom and the main cable, and so on. The results of the excitation experiment with the ambient environment were as close as possible and finally established In the large-scale bridge structure of the finite element model to amend the practical method to serve the bridge of health monitoring and status assessment.

\section{Project background}

Learning eight way self-anchored suspension bridge is located in Nanjing Chunhua Town, and the bridge is 160 meters long. The bridge across the Nanjing-Hangzhou

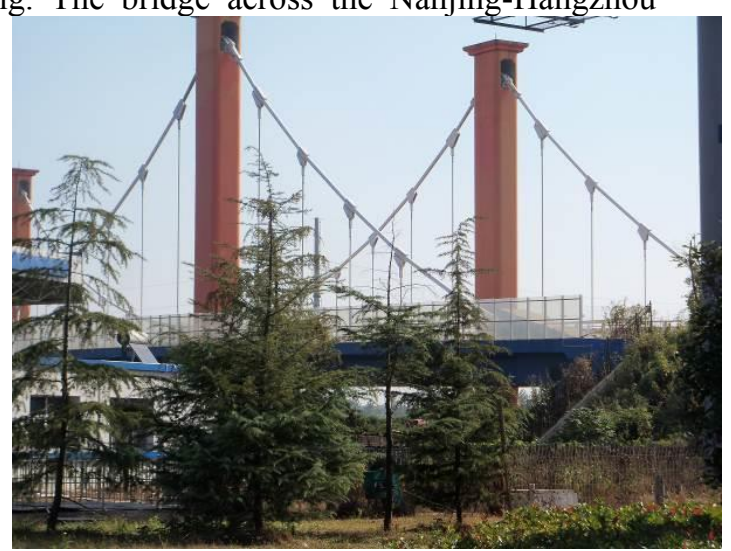

(a) The view under the bridge

Figure 1 Learning eight way self - anchored suspension bridge

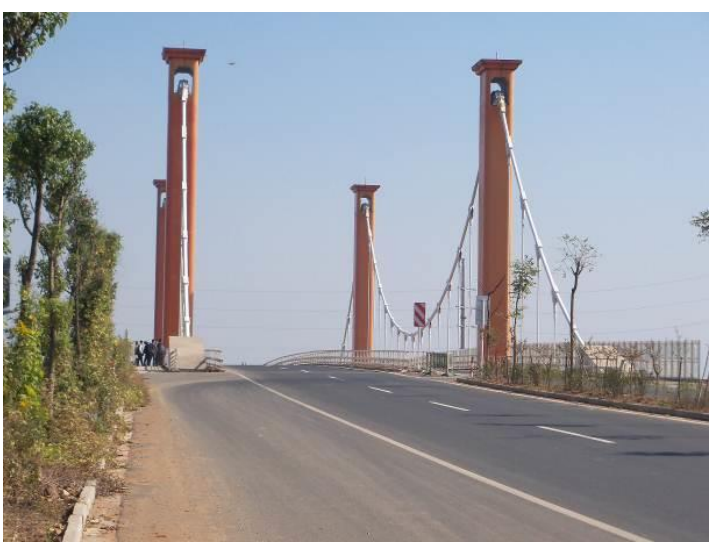

(b) The view on the bridge

calculation model at the same time, and the components with similar mechanical properties are simulated to improve the accuracy of the numerical model. The main cable and the boom are modeled by the rod unit. The bridge deck and the pagoda are modeled by hexahedral solid elements. The total number of points in the model is 67216 , the total number of units is 40572 , and the number of hexahedral entities is 40188 . The number of boom units is 394 , the connection between the main cable and the boom. The main cable and the bridge deck and between the boom and the bridge deck is connected by a JOIN connection, and the hinge connection 
established by such a connection restrains the same line displacement. The finite element model of this paper does not take into account the contribution of the following parts of the bridge pile. The degree of freedom of the bottom of the pier is all constrained, and the side span and the platform are connected by simply supporting the beam. In order to make the calculation result more accurate, the calculation model simulates the true box girder structure of the bridge, as shown in Figure 3 (the finite element model) and Figure 4 (boom number diagram). As the suspension tension of the suspension bridge has great influence on the linear and vibration characteristics of the bridge, the cable force of the test is applied to the corresponding boom by the cooling method in order to ensure the reliability of the calculation results. Figure 5 shows the measured cable force of the learning eight-way self-anchored suspension bridge. Since the test is to use the vibration method to measure the cable force of the boom, the four rigid boom cable forces near the abutment can not be accurately determined. The design boom power is still used in the model.

\section{Modal parameter identification}

Bendat et al. [9] published the "correlation and spectral analysis engineering application" in detail the peak picking method (Peak Picking, PP). The peak method based on the structure of the frequency response function at the natural frequency will have a peak principle. When the output response is used, the frequency spectrum function is used to replace the frequency response function. The modal characteristic frequency is determined by

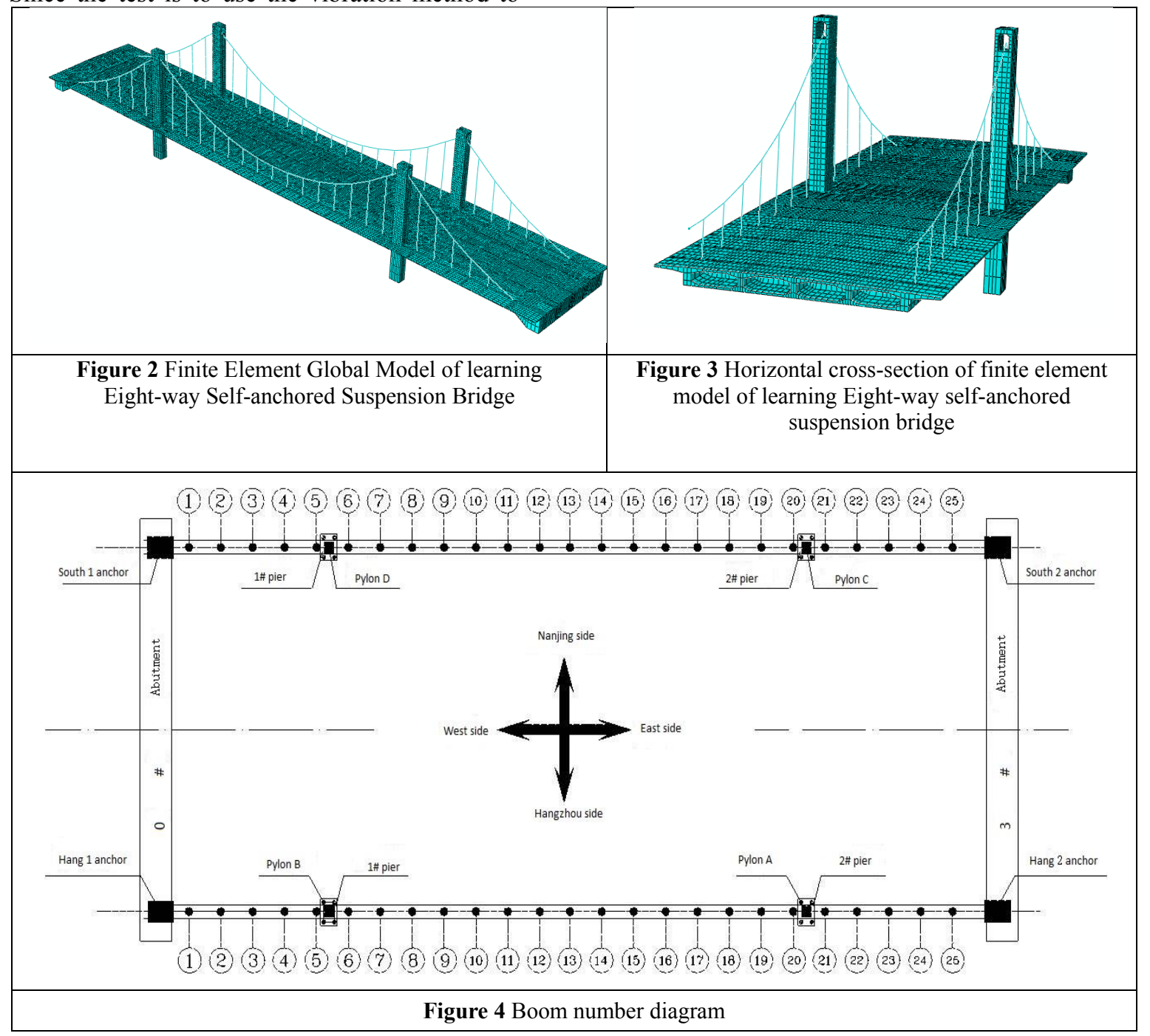




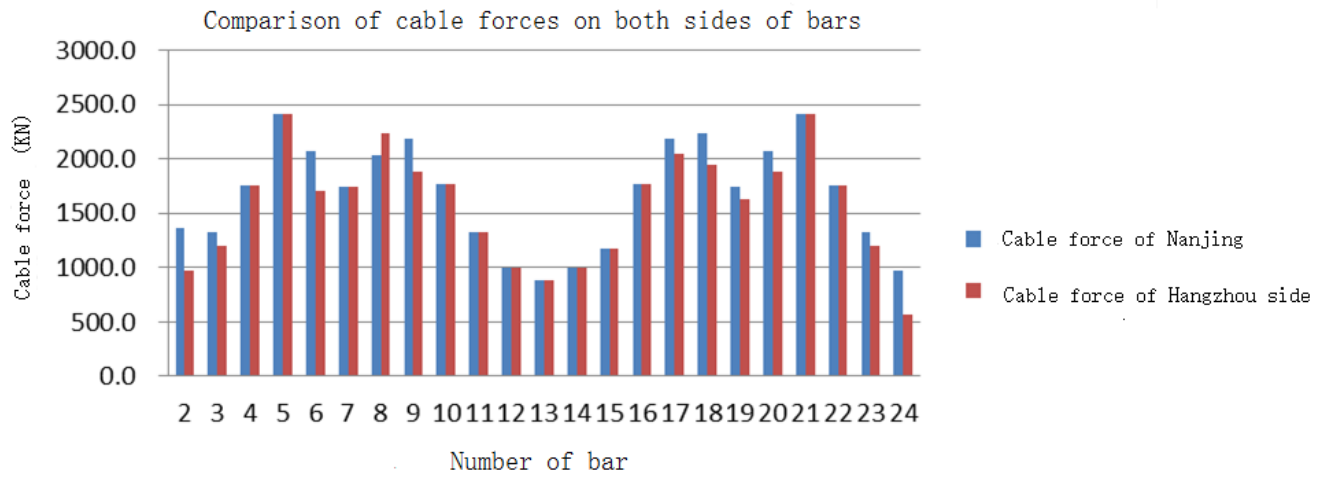

Figure 5 Boom cable force distribution map

The peak of the average regularized power spectral density curve. The modal damping ratio is determined by the half power band method.

\subsection{The basic theory of peak method}

For the multi-degree of freedom damping system, the dynamic equation of the system vibration is:

$$
M(t)+C x(t)+K x(t)=F(t)
$$

Laplace transformation can be converted into

$$
H(s)=\frac{X(s)}{F(s)}=\left[M s^{2}+C s+K\right]^{-1}
$$

$H(s)$ called the system transfer function. If $s=j \omega$, the transformation process is equivalent to the Fourier transform process, the transfer function $H(s)$ is the frequency response function:

$$
\left.H(s)\right|_{s=j \omega}=H(j \omega)=H(\omega)=\frac{1}{K-M \omega^{2}+j \omega C}
$$

For a real-mode system, the frequency response function is further decomposed by Equation (3) :

$$
H_{i k}(\omega)=\frac{x_{i}}{f_{k}}=\sum_{r=1}^{N} \frac{\phi_{i r} \phi_{k r}}{k-\omega^{2} m_{r}+j \omega c_{r}}=\sum_{r=1}^{N} \frac{\phi_{i r} \phi_{k r}}{\left(j \omega-\lambda_{r}\right)\left(j \omega-\bar{\lambda}_{r}\right)}
$$

Where $\mathrm{N}$ is the order of the modal, $\phi_{i r}$ and $\phi_{k r}$ are the modes vector at the $\mathrm{i}$ and $\mathrm{k}$ points of the r-order modal, respectively. $\lambda_{r}$ and $\bar{\lambda}_{r}$ are the conjugate eigenvalues of the structure, respectively.

The dynamic displacement response $x_{i}(\omega)$ of any point $\mathrm{i}$ on the structure can be expressed by the k-point excitation force $f_{k}(\omega)$ and the structural frequency response function $H_{i k}(\omega)$ :

$$
x_{i}(\omega)=\sum_{k=1}^{m} H_{i k}(\omega) f_{k}(\omega)
$$

For environmental excitations, it can be approximated as a white noise signal with uniformly distributed frequency energy. It means that the power spectral density function is uniform distribution over all frequency ranges, so the input at each location satisfies the condition:

$$
f_{k}(\omega)=f(\omega)=C_{1}
$$

$C_{1}$ is a constant. Thus, equation (6) can be simplified as:

$$
x_{i}(\omega)=f(\omega) \sum_{k=1}^{m} H_{i k}(\omega)=C_{1} H_{i}(\omega)
$$

As can be seen from the above equation, the response of the structure $x_{i}(\omega)$ and the total frequency response function of the structural system are equivalent, so the natural frequency of the structure can be obtained directly from the corresponding spectrum $x_{i}(\omega)$.

Select a point of the structure as a reference point, the transfer rate between the response point and the reference point was established to identify the modal vibration of the structural system. The identified vibration mode is not the true mode of the structure, but the work deflection of the structure. It need to set a point which has a clear response in all the frequency as the reference point. The transfer rate between the response point and the reference point can be expressed as:

$$
\alpha_{i}(\omega)=\frac{x_{i}(\omega)}{x_{p}(\omega)}
$$

The sequence $\alpha_{i}(\omega)$ is the working deformation of the frequency $\omega$ corresponding to the structure, and it can be regarded as the modal shape of the system. Equation (4) can be taken into the above formula, obtain:

$$
\alpha_{i}(\omega)=\frac{x_{i}(\omega)}{x_{p}(\omega)}=\frac{f(\omega) \sum_{k=1}^{m} H_{i k}(\omega)}{f(\omega) \sum_{k=1}^{m} H_{p k}(\omega)}=\frac{\sum_{k=1}^{m} H_{i k}(\omega)}{\sum_{k=1}^{m} H_{p k}(\omega)}
$$

Assuming that the solid modes of the structures can be effectively separated from each other and there is no coupling or only weak coupling between them, the system response at the rth order natural frequency is dominated by the vibrations of the rth order mode. Equation (4) and equation (9) can be simply written as:

$$
\alpha_{i}(\omega)=\frac{\frac{\phi_{i r}}{\left(j \omega-\lambda_{r}\right)\left(j \omega-\bar{\lambda}_{r}\right)} \sum_{k=1}^{m} \phi_{k r}}{\frac{\phi_{p r}}{\left(j \omega-\lambda_{r}\right)\left(j \omega-\bar{\lambda}_{r}\right)} \sum_{k=1}^{m} \phi_{k r}}=\frac{\phi_{i r}}{\phi_{p r}}
$$

In the above equation, since the point $p$ is fixed as a reference point, it is a constant value $\phi_{p r}$ for a certain natural frequency $\omega_{r}$. So the above equation can be simplified as:

$$
\alpha_{i}(\omega)=\frac{\phi_{i r}}{\phi_{p r}}=C_{2} \phi_{i r}
$$


From the above equation, the modal mode of the structure at the frequency ${ }^{\omega_{r}}$ can be directly read the transfer rate curve $\alpha_{i}(\omega)$ to obtain. The magnitude of the mode is obtained from the amplitude of the transmission rate at the natural frequency, and the direction of the mode at the frequency or the real part of the transfer rate can be obtained by the crossfire spectrum.

\subsection{Modal Parameter Identification of Suspension Bridge Deck}

The above-mentioned theory shows that the modal parameters can be obtained under the condition that the excitation is unknown, by estimating the self-power spectrum and the cross-power spectrum between the measured points, combining the phase and the transfer rate. Because the environmental excitation is close to the white noise excitation in a certain frequency band, the grid structure is linear small damping vibration under the environment excitation. Based on the assumption of the peak method, the vibration response of the reference
Figure 6 Design of Measuring Points for Modal Recognition of Deck System

(2) Create a data file and import the acquired modal data. There were seven measurement points in the modal analysis, where $\mathrm{C} 4$ was taken as the base point. The collected data were imported into three batches, and each node of the model was assigned. The real value of the measurement is given to the measuring point. For non-measuring points, it is interpolated by the measuring point value, see Figure 6 and Figure 7.

(3) FFT transformation of the data. Since the data acquisition time of each measuring point in the modal test was about $1800 \mathrm{~s}$, and the sampling frequency was $50 \mathrm{~Hz}$, so the total data were about 90000 points. Therefore, the size of each FFT data was 8192 (213), and the signal leakage can be effectively reduced by Hanning window (hanning) function for window processing. It is possible to improve the accuracy of spectral estimation, increase the average number of times, reduce the variance and make full use of the start and end data of the segment by using the overlap processing and averaging processing in the spectrum analysis. The overlap ratio was set for $50 \%$, and the processing was selected linear averaging processing. After FFT

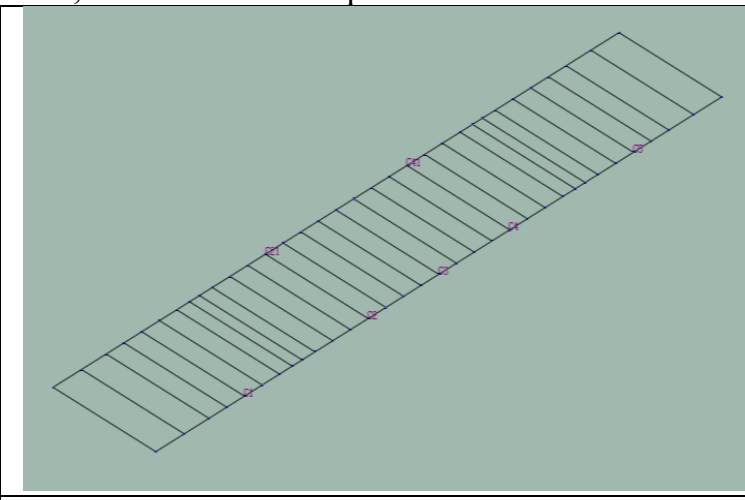

point can be used as input excitation. In this way, the frequency response function can be directly replaced by the self-power spectrum and the cross-power spectrum of the vibration response to estimate the parameters such as frequency, damping and mode. According to the identification method of the peak method, the cantilever bridge deck modal parameters can be identified only by the vibration response of the bridge deck.

(1) Construction of the suspension bridge deck model. According to the analysis, the bridge deck composed of the stiffening beams was the main force component of the self-anchored suspension bridge. The structure of the box girder composed of the deck system is directly related to the overall safety of the whole bridge. The change of the internal force of the boom and the local damage of the box girders directly lead to the change of the vibration characteristics of the deck system. Therefore, this paper only studies the vibration characteristics of the deck system. As the transverse stiffness of the deck system is much larger than the vertical stiffness, vibration is mainly expressed in vertical vibration. According to the principle of less and fine, the measurement points on the deck system are as follows.

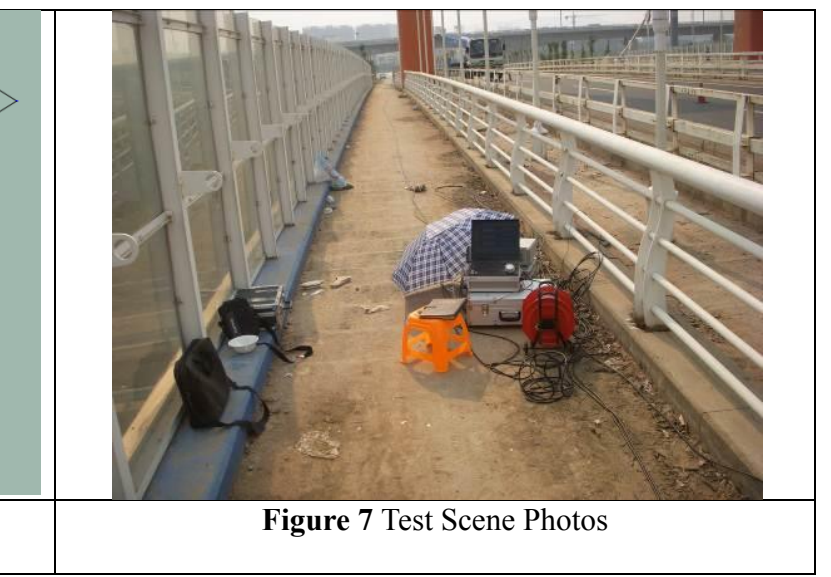

transformation of the collected data, we can get the modal parameters of the self-power spectrum amplitude, phase and coherence function to identify the modal parameters of the system, as shown in Figure 8.

(4) Carry out the discrimination of the real mode. The screening of frequency was subjective, and the above frequency of screening with a lot of false patterns were also identified. It need to be identified. Because the frequency and mode of the multi-degree of freedom system are the characteristic relations of eigenvalues and eigenvectors in mathematical problems. According to the vibration analysis theory, the vibration vector of the same order is completely related, and the vibration vector of different order should be unrelated. This principle can be used as a tool for modal model validation. The modal confidence factor matrix can be constructed. If it is true, the non-diagonal elements in the matrix should be close to zero. According to these criteria, the natural frequencies of vibration in the vertical direction of the bridge are $1.44 \mathrm{~Hz}$ and $3.09 \mathrm{~Hz}$. The learning eight-way self-anchored suspension bridge deck vertical vibration mode which was identified out can be seen in Figure 9. 


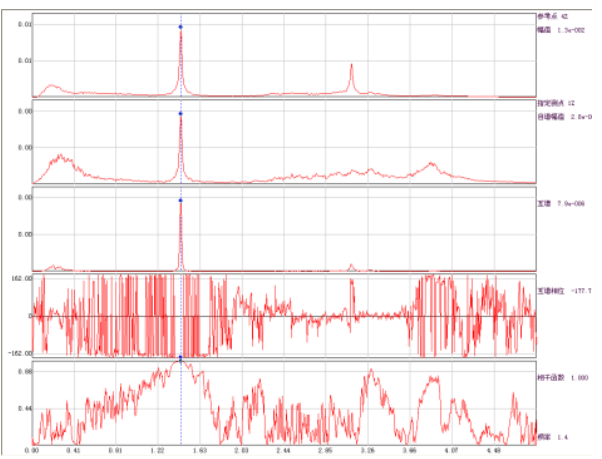

(a) C1 Point spectrum analysis

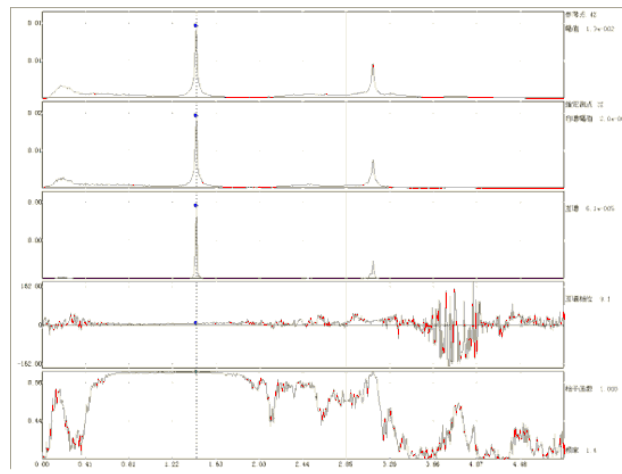

(c) C3 Point spectrum analysis

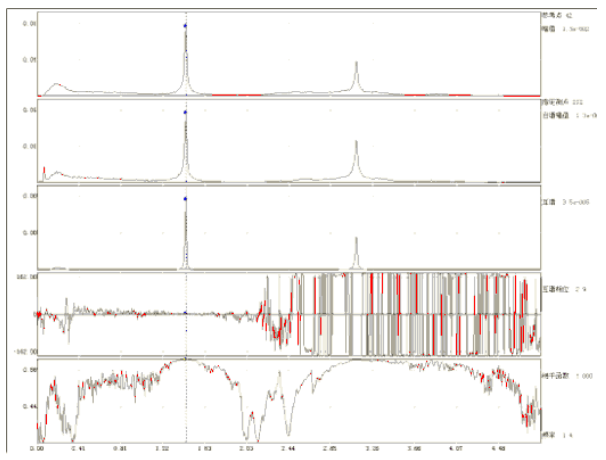

(e) C21 Point spectrum analysis

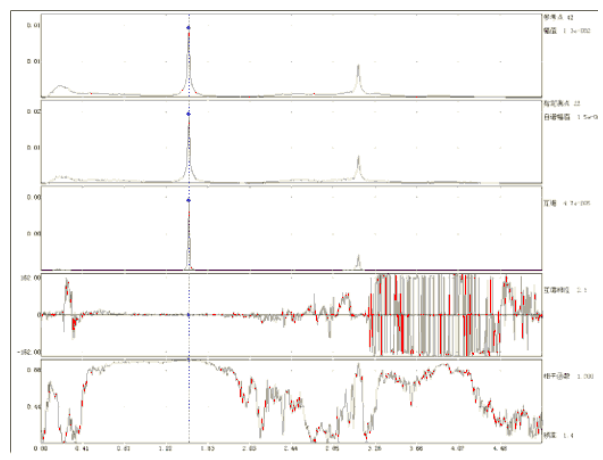

(b) C2 Point spectrum analysis

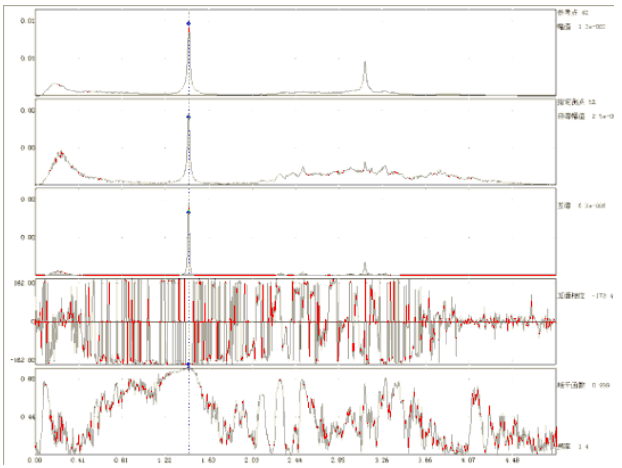

(d) C5 Point spectrum analysis

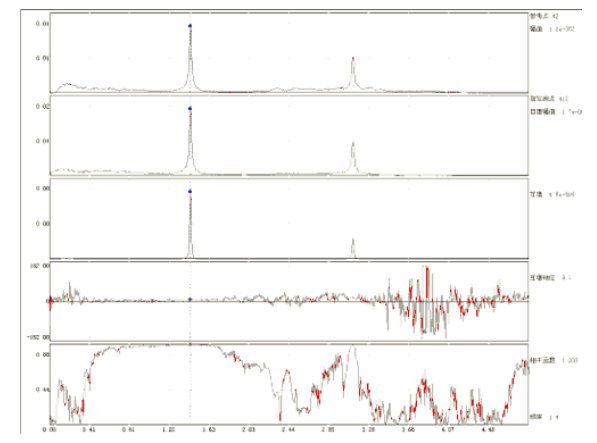

(f) C41 Point spectrum analysis

Figure 8 The self-power spectrum amplitudes, phases and coherence function curves between each measuring points and reference points

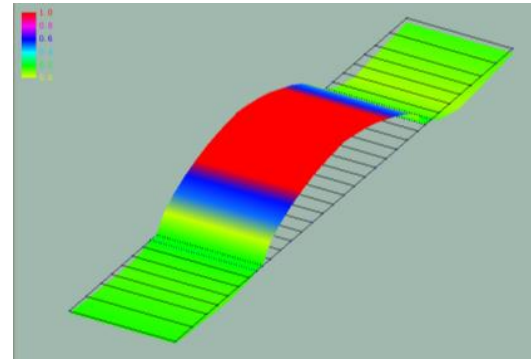

(a) Deck $1.44 \mathrm{~Hz}$ corresponding to the vibration

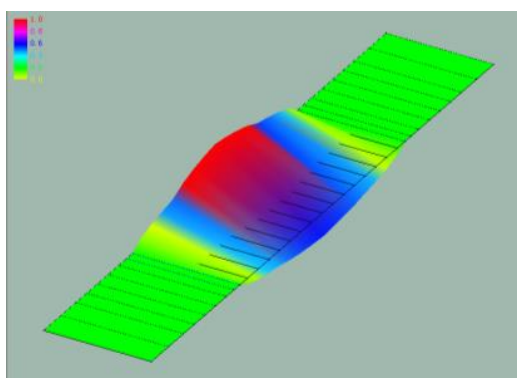

(b) Bridge diagram of $3.09 \mathrm{~Hz}$ corresponding to the vibration vibration pattern

vibration characteristics of the structure in the event of free vibration. It usually includes parameters such as natural frequency, natural mode, modal mass, modal stiffness and modal damping ratio. The most important parameters are the frequency, vibration mode and damping ratio. For the damping system, they are the natural frequency and vibration mode. The main reason
The analysis of the dynamic characteristics of the structure can be also called modal analysis of the structure. The modal of the structure refers to the basic

\section{Finite Element Simulation Analysis of Bridge Vibration Characteristics}


for the analysis of the self-vibration characteristics of the structural system is as follows: 1 . the natural frequency and mode of the structure are an inherent property of the structure itself. It depends only on the stiffness and mass distribution of the structure itself. Therefore, the vibration force and vibration mode of the structure can be used to analyze the stiffness of the structure and the distribution rationality of the stiffness and mass. 2 . During the forced vibration, the correlation between the internal force and the displacement of the structure and the vibration frequency and vibration form of the structure is closely related. The natural frequency and vibration mode are the main parameter to calculate the forced vibration. 3. Therefore, analyzing the natural frequency and vibration mode is the basis of the dynamic response analysis of the structure. The finite element analysis of this bridge testing project will be carried out on commercial software ABAQUS. The pre-processing function of ABAQUS is relatively wonderful, and the function to solving non-linear problems is also better.

\subsection{Numerical model}

In this numerical modeling, the fishbone model [10] is used to simulate the real situation of the suspension bridge. The so-called fishbone model is used in the calculation model, which simulates the components with similar mechanical properties and improves the calculation accuracy of numerical model. The main cable and the boom are modeled by the rod element. The bridge deck and the pagoda are modeled by the hexahedral solid element. The total number of points is 67216 , the total number of the units is 40572 , the number of hexahedral entities is 40188 , and the number of boom units is 394, as shown in Figure 10. Material parameters are in accordance with the relevant norms of value.

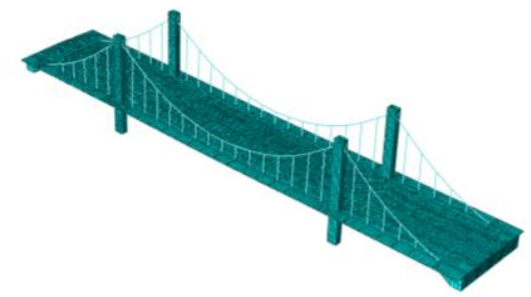

Figure 10 Finite Element Calculation of Integral Model of learning Eight-way Suspension Bridge

In order to make the calculation result more accurate, the calculation model simulates the true box girder

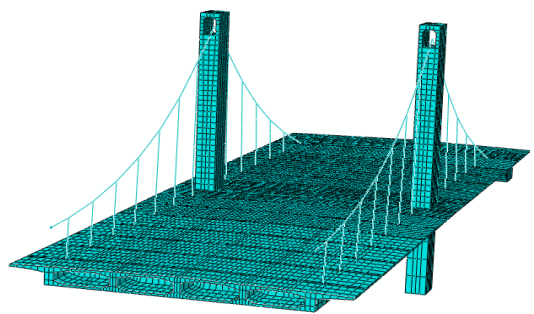

Figure 11 The calculation model transverse section of learning eight-way suspension bridge

\subsection{Finite element analysis}

The first-order natural frequency of the bridge deck is $1.17 \mathrm{~Hz}$, and the analysis result of the self-interacting modal is $1.44 \mathrm{~Hz}$, which means that the error is $18.8 \%$. The first-order mode is shown in Fig. 13, and the results are consistent with modal analysis results. The second-order natural frequency is $2.45 \mathrm{~Hz}$, and the error is $20.7 \%$ comparing with the result of modal analysis $3.09 \mathrm{~Hz}$. The second-order mode is shown in Figure 14, which is consistent with the result of modal analysis. The structure of the bridge, as shown in Figure 11 and Figure 12.

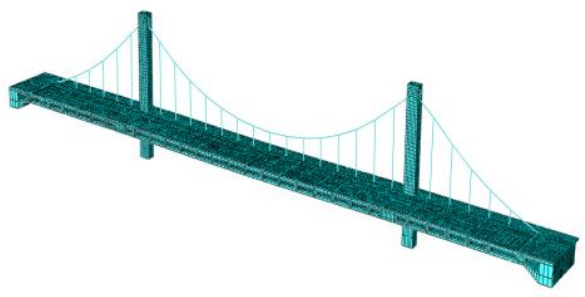

Figure 12 The calculation model longitudinal section of learning eight-way suspension bridge third and fourth order natural frequency are $3.14 \mathrm{~Hz}, 3.61$ $\mathrm{Hz}$, respectively, corresponding to the vibration mode as shown in Figure 15 and Figure 16. The two modes are not self-phantom modal analysis identified, indicating that the actual vibration of the environment did not stimulate the excitation of the bridge of these modes. As the actual material parameters of the bridge need to be obtained by the relevant experiments, the calculation of the material parameters of the model are in accordance with the relevant norms. The calculated natural frequency is not so accurate. 


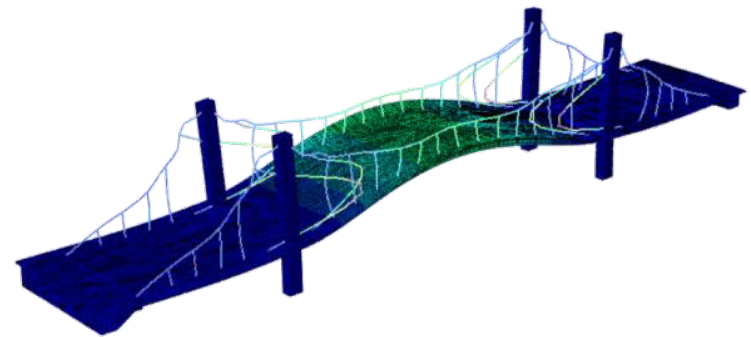

Figure 13 The first-order mode diagram

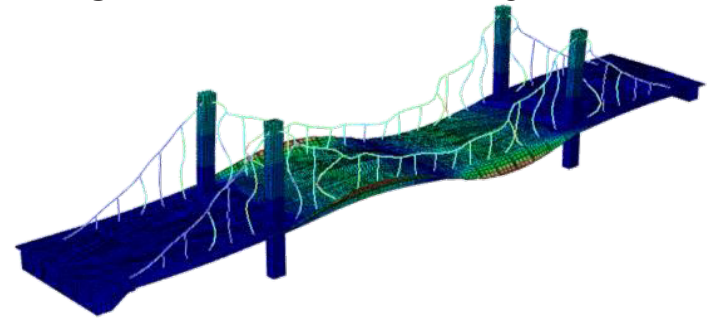

Figure 15 The third-order mode diagram

\section{Conclusion}

Using field testing and finite element simulation, the paper shows the below results: 1) The fishbone model is used to simulate the real situation of the suspension bridge. 2) Peak method is simple and rapid, which have clear physical meaning and is suitable for analysis the modal parameters of the structure under the excitation environment. It can obtain more accurate frequency and vibration results. 3) Using the optimized method to modify the frequency of the grid, the revised design parameters can be well corresponding to the actual state of the grid. The corresponding finite element model reflects the dynamic characteristics of the grid more truly. The modified model can be used as a reference model for the vibration reduction of the grid.

Due to the complex structure of the bridge and the cumbersome material used in the construction process, a good model correction process must be based on the accurate data and the actual material parameters obtained from the experiment. This is the key to the success of the model correction technique and the direction of further study.

\section{References}

1. Miao Chang-qing, Han Hui-ting, Li Ai-qun et al. Analysis on the Original SHM System of Jiangyin Bridge [J]. Journal of Highway and Transportation Research and Development, 2007, 24 (11): 81-86.

2. Zhou Wen-song, Li Hui, Ou Jin-ping et al. Design Method of Data Acquisition System of Health Monitoring Systems for Long Span Bridges [J]. Journal of Highway and Transportation Research and Development, 2006, 23 (3): 83-87.

3. Ji Wei-hong, Song Yu-pu, Liang Bing. Numerical Simulation of Damage Diagnosis of Space Truss Structures by ANSYS [J]. Journal of Wuhan University of Technology (Traffic Science and Engineering), 2006 (2): 285-288.

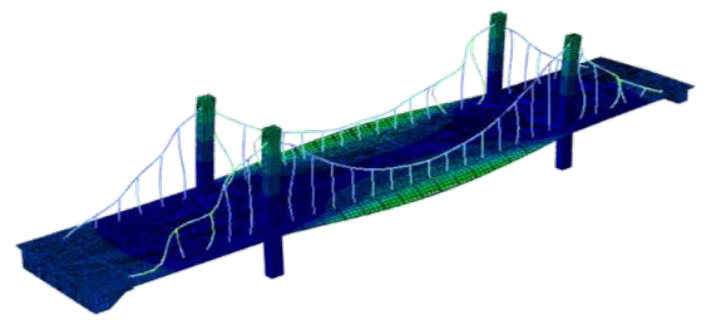

Figure 14 The second-order mode diagram

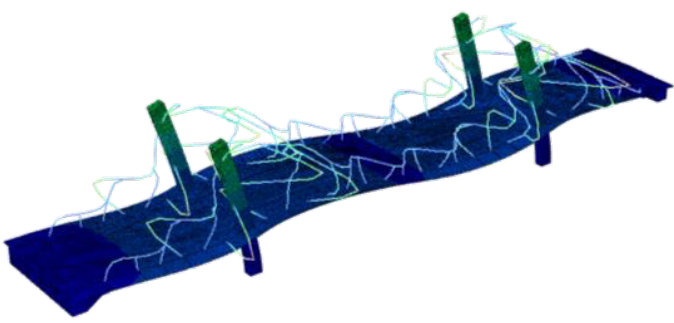

Figure 16 The fourth-order mode diagram

4. Gao Wei-cheng, Liu Wei. Numerical Simulation and Experimental Study on Damage Identification of Reticulated Shell [J]. Engineering Mechanics, 2006 (10): 111-117.

5. Wang Hao, Wang Fu-quan, Li Ai-qun et al. Multi-phase FE Model Updating on Long-span Cable-supported Bridges [J]. Engineering Mechanics, 2009, 26 (10): 111-117.

6. Jaishi B, Ren W X. Structural finite element model updating using ambient vibration test results [J]. Journal of Structural Engineering, 2005, 131(4): 617-628.

7. Li Zhao-xia, Li Ai-qun, Chen Hong-tian, et al. Finite Element Modeling for Health Monitoring and Condition Assessment of Long-Span Bridges [J]. Journal of Southeast University: Natural Science Edition, 2003, 33 (5): 562-572. (in Chinese)

8. Ren Wei-xin, Chen Hua-bin. Response-Surface Based on Finite Element Model Updating of Bridge Structures [J]. China Civil Engineering Journal, 2008, 41 (12): 73-78. (in Chinese)

9. Bendat J. S. Piersol A. G. Engineering applications of correlation and spectral analysis [M]. New York: Wiley-Inter science, 1980. (Peak Value Method)

10. Pei Xing-zhu, Liu Zheng-wei, Zhou Xiao-shong. Basic research on fishbone vibration model of double wings [J]. Journal of Jiangsu University of Science and Technology: Natural Science Edition, 2007, 06 . 